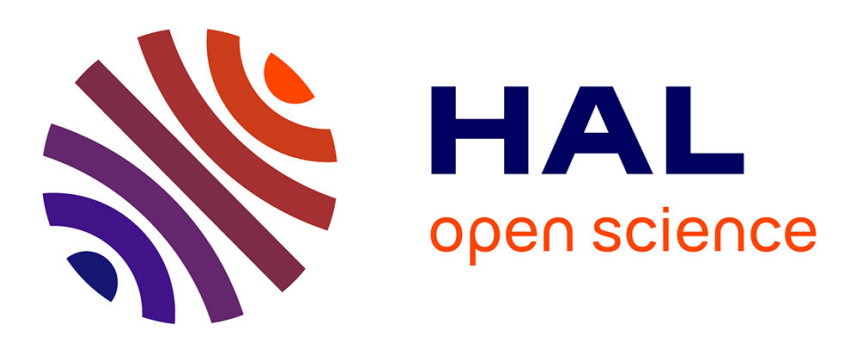

\title{
Détermination des constantes scalaires de l'état de base de SF 6
}

\author{
H. Berger, A. Aboumajd, R. Saint-Loup
}

\section{To cite this version:}

H. Berger, A. Aboumajd, R. Saint-Loup. Détermination des constantes scalaires de l'état de base de SF 6. Journal de Physique Lettres, 1977, 38 (18), pp.373-375. 10.1051/jphyslet:019770038018037300 . jpa-00231399

\section{HAL Id: jpa-00231399 https://hal.science/jpa-00231399}

Submitted on 1 Jan 1977

HAL is a multi-disciplinary open access archive for the deposit and dissemination of scientific research documents, whether they are published or not. The documents may come from teaching and research institutions in France or abroad, or from public or private research centers.
L'archive ouverte pluridisciplinaire HAL, est destinée au dépôt et à la diffusion de documents scientifiques de niveau recherche, publiés ou non, émanant des établissements d'enseignement et de recherche français ou étrangers, des laboratoires publics ou privés. 


\title{
DÉTERMINATION DES CONSTANTES SCALAIRES DE L'ÉTAT DE BASE DE SF 6
}

\author{
H. BERGER, A. ABOUMAJD et R. SAINT-LOUP
}

Laboratoire de Spectronomie Moléculaire (*), Université de Dijon, 6, boulevard Gabriel, 21000 Dijon, France

(Reçu le 1er juillet 1977, accepté le 18 août 1977)

\begin{abstract}
Résumé. - A partir de l'analyse de la bande Raman $v_{2}$ de $\mathrm{SF}_{6}$, les constantes scalaires de l'état de base ont pu être déterminées :

$$
B_{0}=0,09111 \pm 0,00005 \mathrm{~cm}^{-1}, \quad D_{0}=(0,16 \pm 0,08) 10^{-7} \mathrm{~cm}^{-1} .
$$

Abstract. - From analysis of the $v_{2}$ Raman band the molecular constants of the ground state of $\mathrm{SF}_{6}$ have been determined :

$$
B_{0}=0.09111 \pm 0.00005 \mathrm{~cm}^{-1}, \quad D_{0}=(0.16 \pm 0.08) 10^{-7} \mathrm{~cm}^{-1}
$$
\end{abstract}

1. Introduction. - Dans cette étude nous abordons pour la première fois la détermination des constantes scalaires de la molécule $\mathrm{SF}_{6}$ dans son état vibrationnel fondamental. La méthode la plus directe pour déterminer ces constantes serait d'analyser le spectre de rotation pure ; or, dans l'état vibrationnel fondamental d'une molécule toupie sphérique $\mathrm{XY}_{4}$ ou $\mathrm{XY}_{6}$, la variation du moment dipolaire ou de la polarisabilité au cours d'une rotation de la molécule est nulle en première approximation. Le spectre de rotation est considéré comme interdit : c'est une propriété générale des systèmes quantiques à symétrie sphérique.

1.1 DÉVELOPPEMENT DU MOMENT DE TRANSITION. L'opérateur de transition $M$ (moment dipolaire ou polarisabilité) est généralement développé en série en fonction des coordonnées normales et des coordonnées de rotation $Q_{\mathrm{R}}$; le développement en fonction de $Q_{\mathrm{R}}$ s'écrit alors :

$M=M^{0}+\left(\frac{\partial M}{\partial Q_{\mathrm{R}}}\right)_{Q_{\mathrm{R}}=0} Q_{\mathrm{R}}+\frac{1}{2}\left(\frac{\partial^{2} M}{\partial Q_{\mathrm{R}}^{2}}\right)_{Q_{\mathrm{R}}=0} Q_{\mathrm{R}}^{2}+\cdots$

Les termes du développement donnent une contribution non nulle seulement si leur symétrie correspond à celle d'une composante du moment $M$, exprimée dans le groupe de la molécule; cette condition peut s'exprimer par :

$$
\Gamma_{Q} n \supset \Gamma_{M}
$$

(*) Equipe associée au C.N.R.S.
Les coordonnées de rotation sont la base de la représentation $D^{(1 \mathrm{~g})}$ du groupe des rotations $O(3)$; la restriction de $\mathrm{D}^{(1 \mathrm{~g})}$ dans le groupe de la molécule $\left(\mathrm{O}_{h}\right)$ est indiquée dans le tableau I.

\section{TABleaU I}

Propriétés de symétrie des opérateurs de transition dans la chaîne de groupes $\mathrm{O}(3) \supset \mathrm{O}_{\mathrm{h}}$.

\begin{tabular}{|c|c|c|}
\hline & $\mathrm{O}(3)$ & $\mathrm{O}_{\mathrm{h}}$ \\
\hline Moment dipolaire & $\overline{D^{(1 u)}}$ & $\overline{F_{11}}$ \\
\hline Polarisabilité & $D^{(08)}+D^{(28)}$ & $A_{1 g}+E_{g}+F_{2 g}$ \\
\hline $\begin{array}{c}\text { Coordonnées } \\
\text { rotation } Q_{\mathrm{R}} \\
\text { Terme en } Q_{\mathrm{R}}^{2}\end{array}$ & $\begin{array}{c}D^{(18)} \\
{\left[D^{(18)} \times D^{(18)}\right]^{0,2 g}}\end{array}$ & $\begin{array}{c}F_{1 g} \\
A_{1 g}+E_{g}+F_{2 g}\end{array}$ \\
\hline
\end{tabular}

[Symmetry properties of the transition operators in the chain of groups $\mathrm{O}(3) \supset \mathrm{O}_{\mathrm{h}}$.]

1.2 TERME D'ORDRE 1. - Il apparaît dans le tableau I, que la condition (2) n'est pas remplie pour le terme du premier degré par rapport à la coordonnée de rotation $Q_{\mathrm{R}}$. Le spectre de rotation Infrarouge ou Raman est donc interdit en première approximation.

1.3 TERME D'ORDRE 2. - A la différence des molécules $\mathrm{XY}_{4}$, les transitions dipolaires électriques sont interdites au $2^{e}$ ordre d'approximation et d'ailleurs à tout ordre du développement (1) en raison de la parité du moment dipolaire : ce dernier est en effet de parité « $u$ » alors que les termes rotationnels du développement (1) sont tous de parité « $\mathrm{g} »$. 
Le spectre Raman de rotation pure n'est pas interdit au second ordre d'approximation mais comme pour les molécules $\mathrm{XY}_{4}$, il sera sans doute difficile de le mettre en évidence. Ainsi, la seule possibilité d'atteindre les constantes de l'état de base des molécules $\mathrm{XY}_{6}$ réside dans l'analyse des spectres de vibrationrotation; il y a 2 méthodes directes pour atteindre ce but ; utiliser :

i) Les transitions permises et interdites d'un spectre IR comme l'ont fait Tarrago et al. pour $\mathrm{CH}_{4}$ [1] ainsi que Pierre et al. pour $\mathrm{SiH}_{4}$ [2].

ii) Les transitions Raman comme l'ont fait Herranz et Stoïcheff [3] pour $\mathrm{CH}_{4}$, Kąttenberg et Oskam pour $\mathrm{SiH}_{4}$ [4] et récemment Brodersen et al. pour $\mathrm{CD}_{4}$ [5].

C'est la seconde méthode que nous avons utilisée pour déterminer les constantes $B_{0}$ et $D_{0}$ de $\mathrm{SF}_{6}$.

2. Spectre Raman de la bande $v_{2}$. - La haute résolution du spectrographe Raman de Dijon $\left(0,03 \mathrm{~cm}^{-1}\right)$ décrit antérieurement $[6,7]$ nous a permis d'enregistrer le spectre Raman- de $\mathrm{SF}_{6}$; les bandes fondamentales $v_{1}\left(\mathrm{~A}_{1 \mathrm{~g}}\right), v_{2}\left(\mathrm{E}_{\mathrm{g}}\right)$ et $v_{5}\left(\mathrm{~F}_{2 \mathrm{~g}}\right)$ ont été observées, mais seule la bande $v_{2}$ peut se prêter à une analyse rotationnelle précise.

En effet pour cette bande, les structures fines, correspondant à deux valeurs de $J$ voisines, ne se superposent pas; le spectre apparaît uniquement avec sa structure en $J$, rappelant celle d'un spectre de rotation pure (ce n'est pas le cas pour la bande $v_{5}$ ).

Sur le spectre de la figure 1 , la branche $Q$ de la bande $v_{2}\left(\mathrm{à} 643,35 \mathrm{~cm}^{-1}\right)$ et quelques bandes chaudes voisines apparaissent saturées. De part et d'autre de la branche $Q$ le spectre présente une structure dont la période est voisine de $4 B_{0}$; elle correspond aux raies $\mathrm{O}$ et $\mathrm{S}$. Les branches $\mathbf{P}$ et $\mathbf{R}$ n'apparaissent pas distinctement.

3. Résultats et discussion. - Pour obtenir des expressions dépendant uniquement des constantes de l'état de base, il suffit de faire la différence des nombres d'onde des transitions aboutissant au même niveau excité. En première approximation nous considérons que l'énergie de l'état de base est donnée par l'expression suivante :

$$
E_{0}=B_{0} J_{0}\left(J_{0}+1\right)-D_{0} J_{0}^{2}\left(J_{0}+1\right)^{2} .
$$

En utilisant les nombres d'ondes des raies $\mathrm{O}$ et $\mathrm{S}$ on obtient la relation suivante :

$\frac{\sigma \mathrm{S}_{J_{0}-2}-\sigma \mathrm{O}_{J_{0}+2}}{8 J_{0}+4}=B_{0}-D_{0}\left(2 J_{0}^{2}+2 J_{0}+8\right)$.

Les données de plusieurs enregistrements de cette bande ont été utilisées pour déterminer les constantes $B_{0}$ et $D_{0}$.

Les valeurs des constantes $B_{0}$ et $D_{0}$ sont suivies de leur intervalle de confiance à $95 \%$ :

$$
\begin{aligned}
& B_{0}=0,09111 \pm 0,00005 \mathrm{~cm}^{-1}, \\
& D_{0}=(0,16 \pm 0,08) 10^{-7} \mathrm{~cm}^{-1} .
\end{aligned}
$$

Au cours de cette étude nous n'avons pas pris en considération le splitting de l'état excité puisqu'il n'est pas observé expérimentalement; néanmoins il peut introduire une asymétrie dans la forme des raies et modifier légèrement la position du centre de la structure rotationnelle. Si l'importance du splitting peut être évaluée à partir de l'opérateur de Coriolis du premier ordre entre les bandes $v_{2}\left(\mathrm{E}_{\mathrm{g}}\right)$ et $v_{5}\left(\mathrm{~F}_{2 \mathrm{~g}}\right)$ $\left(\zeta_{25}=1\right)$, l'importance de l'asymétrie dans la forme des raies nous semble difficile à évaluer et de ce fait l'incertitude indiquée ci-dessus est uniquement statistique.

Jusqu'alors, la valeur de $B_{0}$ était calculée à partir de la distance interatomique S-F obtenue dans une expérience de diffraction électronique par Ewing et Sutton [6] $(\mathrm{S}-\mathrm{F}=1,564 \pm 0,010 \AA)$; la valeur de $B_{0}$ déduite de cette mesure était égale à

$$
0,0906 \pm 0,0011 \mathrm{~cm}^{-1} \text {. }
$$

La valeur de $B_{0}$ a donc été précisée de façon significative et pour la première fois la constante $D_{0}$ a été évaluée.

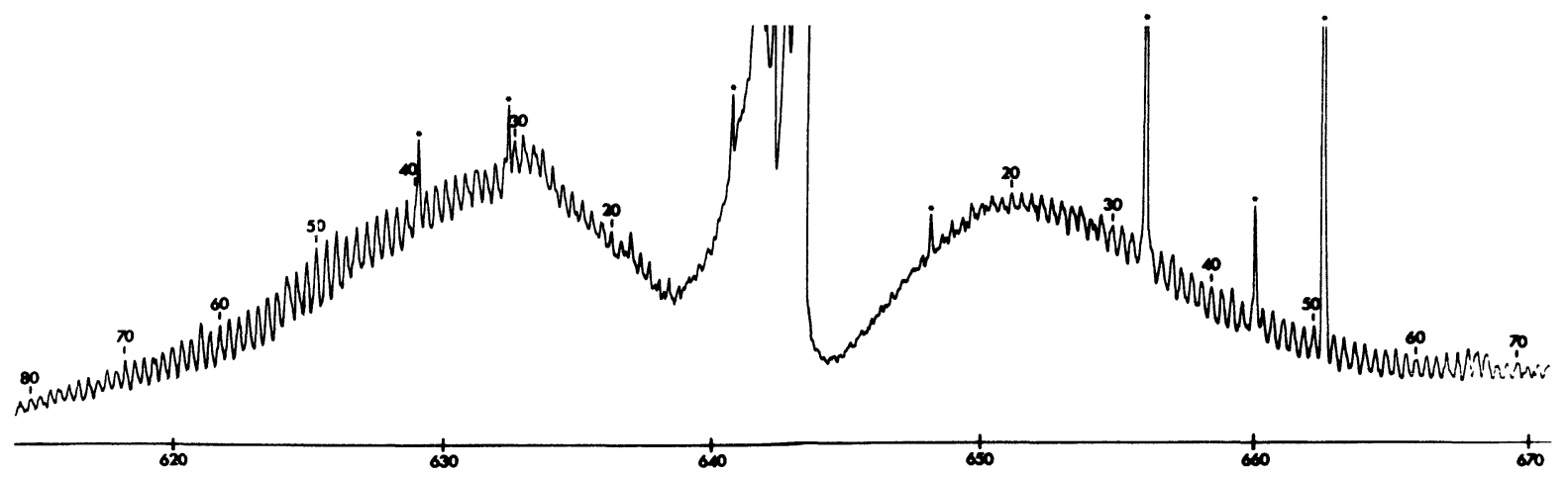

FIg. 1. - Spectre Raman de la bande $v_{2} \mathrm{de} \mathrm{SF}_{6}$. Le nombre $J$ caractérisant l'état de base des transitions est indiqué au sommet de quelques raies. Les raies marquées d'un astérisque correspondent au spectre du thorium, utilisé pour l'étalonnage.

[Raman Spectrum of the $v_{2}$ band of $\mathrm{SF}_{6}$. The $J$ numbering of the ground state is indicated at the top of some lines. The lines with an asterisk belong to the thorium spectrum used for the calibration.] 


\section{Bibliographie}

[1] Tarrago, G., Dang Nhu, M., Poussigue, G., Guelachvili, [5] Brodersen, S., Gray, D. L. et Robiette, A. G. (à paraître G. et Aміот, C., J. Mol. Spectrosc. 57 (1975) 246.

[2] Pierre, G., Guelachvili, G. et Амiot, C., J. Physique 36 (1975) 487.

[3] Herranz, J. et Stoicheff, B. P., J. Mol. Spectrosc. 10 (1963) 448.

[6] Moret-Bailly, J. et Berger, H., C.R. Hebd. Séan. Acad. Sci. 269 (1969) 416.

[7] Berger, H., Faivre, M., Champion, J. P. et Moret-Bailly, J., J. Mol. Spectrosc. 45 (1973) 298.

[4] Kattenberg, H. W. et Oskam, A., J. Mol. Spectrosc. 49 (1974) [8] Ewing, V. C. et Sutton, L. E., Trans. Faraday Soc. 59 (1963) 52. 1241. 